\title{
Automated stirring device for continuous stirring while sampling in liquid chromatography systems
}

Omer Markovitch ${ }^{1,2, *}$, Jim Ottelé ${ }^{2}$, Obe Veldman $^{3}$, and Sijbren Otto ${ }^{2}$

1 Origins Center, Groningen, The Netherlands

2 Center for Systems Chemistry, Stratingh Institute, University of Groningen, Groningen, The Netherlands

3 Veldman Technische Ontwikkeling en Advisering, Groningen, The Netherlands

* omermar@gmail.com

\section{ABSTRACT}

A device is presented, which enables continuous stirring of samples whilst inside an ultra-performance liquid chromatography system. Utilizing standard magnetic stirring bars that fit standard vials, the device allows for the automation of experimental setups that require stirring. The device is designed such that it can replace the standard sample holder and fits in its place, while being battery operated. The use of 3D printing and commercially available parts enables low-effort and low-cost device production, as well as easy modifications. Various tests were performed by following the kinetics of a dynamic combinatorial library that is known for exhibiting self-replication under mechanical agitation, via fiber growth-breakage mechanism. Design files and schematics are available. 
High- and ultra- performance liquid chromatography (HPLC and UPLC, respectively) are common analytical tools for the detection and identification of components in complex mixtures ${ }^{1}$. At the heart of these techniques is sampling and then separation of the sample by the use of a column with appropriate properties, leading to different compounds eluting at different times from the column.

Stirring a sample is fundamental in many experimental setups as it promotes mixture homogeneity. Furthermore, in systems where large assemblies are formed, mechanical agitation can lead to their breakage and is an important part of the system's behavior ${ }^{2,3}$. Stirring can be done by placing a (Teflon coated) magnetic bar within a sample and placing the sample over a device with a rotating magnetic field. Here, we have developed a UPLC stirring device that replaces a standard sample holder and enables battery-powered magnetic stirring in a similar manner to standard laboratory stirring devices. Any experimental setup that requires continuous stirring can now be run inside the UPLC machine, allowing for multiple measurements at various times without the need for sample preparation or human intervention. We demonstrate the applicability of the device with the usage of a system that has previously been shown uniquely respond to stirring - exhibiting exponential growth that is enables through a fiber elongation/breakage mechanism ${ }^{4,5}$.

The development of this device employed open-source content and 3D printing, aligned with the increased do-it-yourself movement in sciences $6,7,8,9$.

\section{Methods}

\section{Design and fabrication of the stirring device}

3D printing was used for maximum versatility in design and manufacturing, as well as to allow userspecific modifications. User requests and stirring readouts are handled via ARDUINO Pro Mini 3.3V $8 \mathrm{MHz}$ microcontroller ${ }^{10}$. A standard motor is used for rotating a plate with magnets (magnet dimensions $25 \times 8 \times 1 \mathrm{~mm}$, see Supplamentary Information (SI)). Control over precise rotation rate of the plate is done via a magnetic sensor.

The device's profile is approximately $4 \mathrm{~mm}$ higher than the standard default sample holder (catalogue number 700005209), and consequently UPLC needle height was adjusted. An overview of the device is given in Fig. 1. 


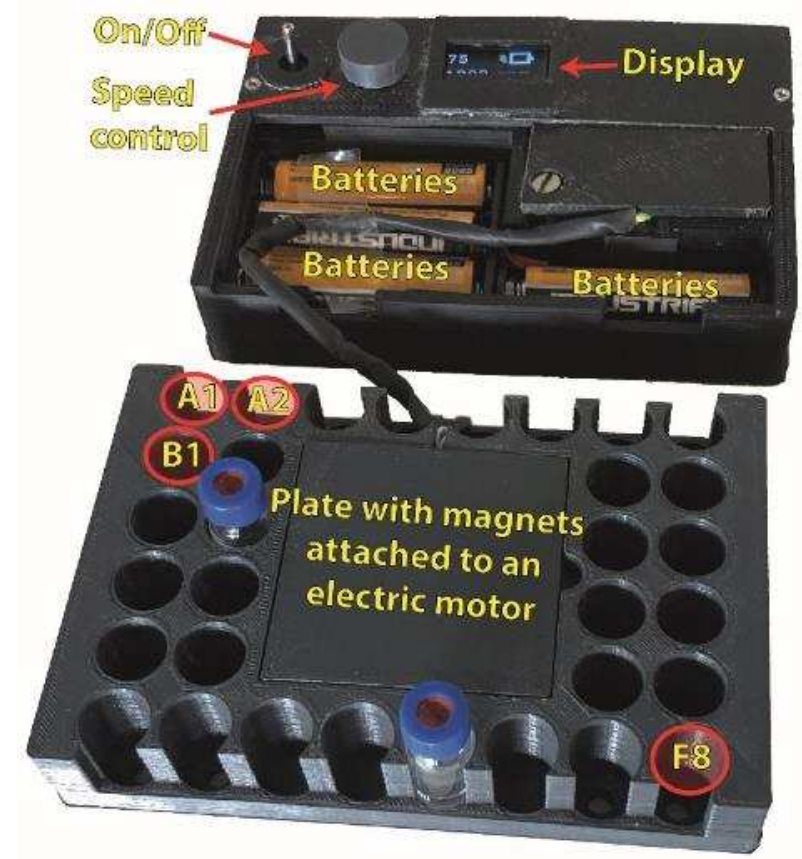

Fig. 1. Device overview. Sample holder positions are labeled A1 to F8.

\section{UPLC sample preparation}

A $2 \mathrm{mM}$ aqueous stock solution was prepared by dissolving $1.2 \mathrm{mg}$ building-block (Fig. 2. Peptide amino acids sequence: Gly-Leu-Lys-Phe-Lys) in $607 \mu \mathrm{L}$ borate buffer (50 mM, pH 8.12). Samples were prepared by adding $250 \mu \mathrm{L}$ of the stock solution to a UPLC vial (dimensions $12 \times 32 \mathrm{~mm}$ ) and diluting it with 750 $\mu \mathrm{L}$ borate buffer. A Teflon coated stirring bar (dimensions $5 \times 2 \mathrm{~mm}$ ) was added to some of the samples and the vials were closed with a Teflon-lined screw cap.

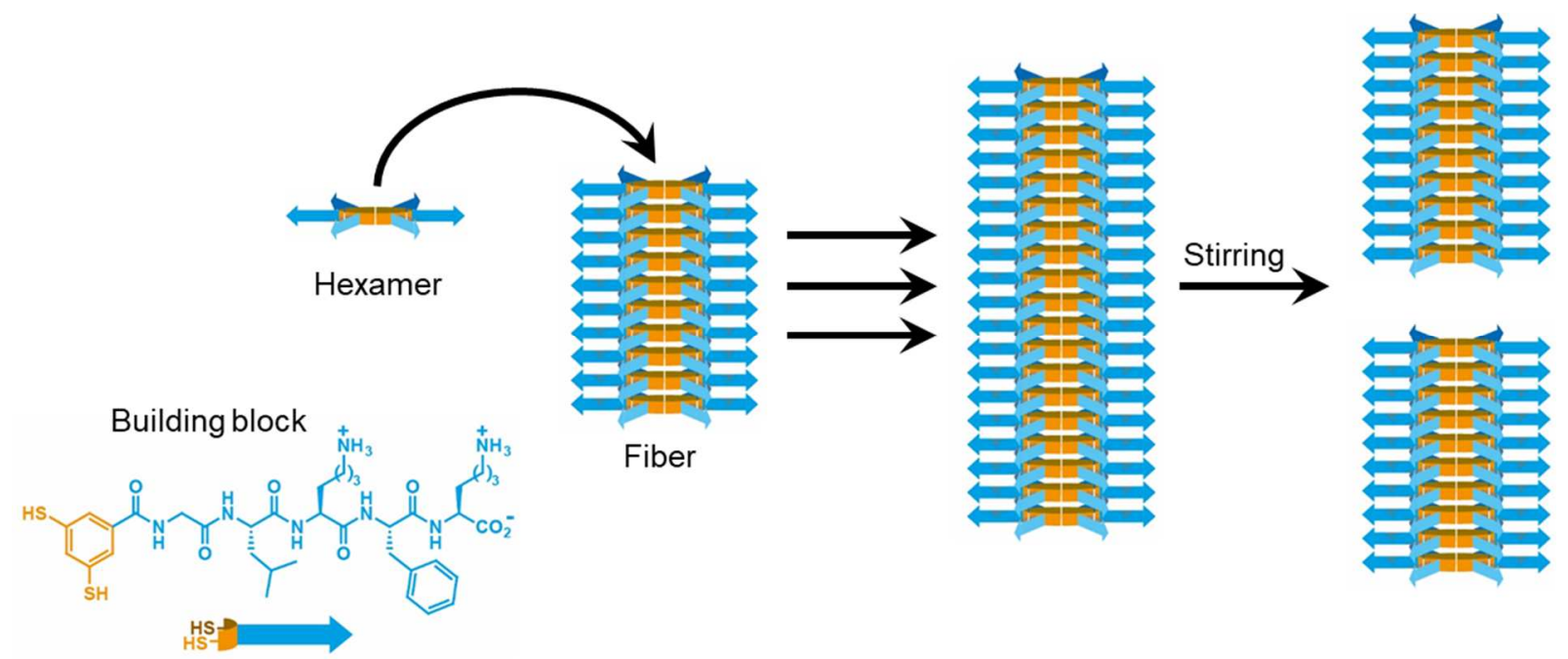

Fig. 2: Chemical system used to probe reproducibility of agitation. Oxidation of the dithiol building block leads to the formation of a mixture of disulfide macrocycles of different ring sizes (not shown). Of these the hexamer self-assembles. The kinetics of autocatalytic formation of hexamer is highly sensitive to agitation, with growth rate depending linearly on the number of fiber ends. ${ }^{5}$ Fibers are fragile and the number of fiber ends is determined by the agitation regime. 


\section{Experiments and measurements}

After preparation, samples were placed within the stirring device inside the UPLC machine, with stirring turned on. The system was monitored by subjecting it to periodic UPLC analysis using a Waters Acquity UPLC-H Class system equipped with a photo diode array detector. All analyses were performed using a reversed-phase UPLC column (Aeris Peptide $1.7 \mu \mathrm{m}$ XB-C18 x $2.10 \mathrm{~mm}$, Phenomenex). The column temperature was kept at $35^{\circ} \mathrm{C}$, and the sample chamber was kept at $40{ }^{\circ} \mathrm{C}$. UV absorbance was monitored at $254 \mathrm{~nm}$. Additional occasional column washes performed. The eluents used in the UPLC separation consist of $\mathrm{H}_{2} \mathrm{O}$ and acetonitrile, both are UPLC grade and contain $0.1 \mathrm{v} \%$ Trifluoroacetic acid. Gradient and peak integration algorithm are given in the SI.

For pre-oxidation experiments, the samples were prepared as described before, with the addition of 0.5 equivalents of sodium perborate $\left(\mathrm{NaBO}_{3}\right)$ so that $50 \%$ of the building-block amount is oxidized. Then, the resulting mixture was kept inside the UPLC sample holder and subjected to periodic UPLC analysis as described above. The samples were stirred using the designed device at 200 and 1000 revolutions per minute (rpm).

Device's position used for samples are C2, C7, D2 and D7 (Fig. 1).

\section{RESULTS AND DISCUSSION}

\section{Design of device}

The device's dimensions are such that it is interchangeable with the manufacturer-provided sample holder. Each vial holder dimension and position exactly match the standard. A motor is used for rotating an internal plate with magnets, such that the magnetic stirring bars inside the sample vials will rotate and thus stirring is achieved. The control module is separated from the stirring module (Fig. 1). The stirring speed range is 200-1200 rpm (in jumps of 100) and is controlled by an onboard microcontroller and an organic light emitting diode screen provides the actual readout of the battery status and rotation rate.

\section{Validation}

To demonstrate the new device, a dynamic system of dithiols is studied (Fig. 2). This system is excellent candidate to validate the device as: (i) the material is heterogeneous and settles to the bottom when unstirred, and (ii) the kinetics are highly sensitive to shear stress, as it has previously been shown that when it is subjected to mechanical agitation hexamer macrocycles assemble into fibers and exponentially grow and undergo self-replication ${ }^{4}$. Initial formation of fiber seeds (nucleation) is a stochastic process, and exponential growth occurs through a fiber elongation-breakage mechanism ${ }^{5}$ (Fig. 2). Due to the system's unique response to stirring, it is chosen for the device's demonstration.

Fig. 3a shows the emergence kinetics of hexamers using the new stirring device, with and without stirring (4 repeats were performed for each case). Indeed, after approximately 100 hours the total percentage of building block mass in the stirred samples reached an average value of $79 \pm 5$ vs. only $13 \pm 5$ when unstirred, demonstrating the device's ability to regularly stir the samples continuously through the course of the experiments. The small differences within each set of repeats is attributed to the stochastic nature of fiber nucleation. High throughput automatic sampling of the device allows for 60 to 90 consecutive measurements per each sample over the course of a week, and is indicated with small black dots on the very top of Fig. 3a. Animation of UPLC traces is given in the SI.

Given the high number of consecutive sampling it is important to test if the sampling quality is consistent throughout the experiments. Fig. 3b shows that, at each time point, the total area of all peaks is well 
conserved throughout, with a relative standard deviation of only $2.27 \%$ and $2.30 \%$, respectively for samples number 3 and 4 .

Fig. 4 shows that indeed stirring at different speeds leads to different rates of the exponential growth, borne by the established role of fibers and their breakage in this process ${ }^{5}$. This is in agreement with previous observations. It is noted that at the elevated temperature experiments were performed here (40 ${ }^{\circ} \mathrm{C}$ ), hexamer formation and replication speed are similar to the speed of BB oxidation, which would make it difficult to observe differences under different stirring rates. Therefore, experiments shown in Fig. 4 were done by first pre-oxidation the building-block solution and then comparing 1000 with $200 \mathrm{rpm}$ stirring rates (see experimental section).

Visual inspection of positions A4, A5, C2, D2, C7, D7, F4 and F5 (Fig. 1) confirmed equal rotation of stirring bar, while positions in columns 1 and 8 exhibit diminished reproducibility of the stirring effect as they further away from the device's center and experience lower magnetic field.

Starting from fresh batteries, the battery level is typically reduced to $35 \%$ after approximately 72 hours of continuous stirring, at which point they were replaced.
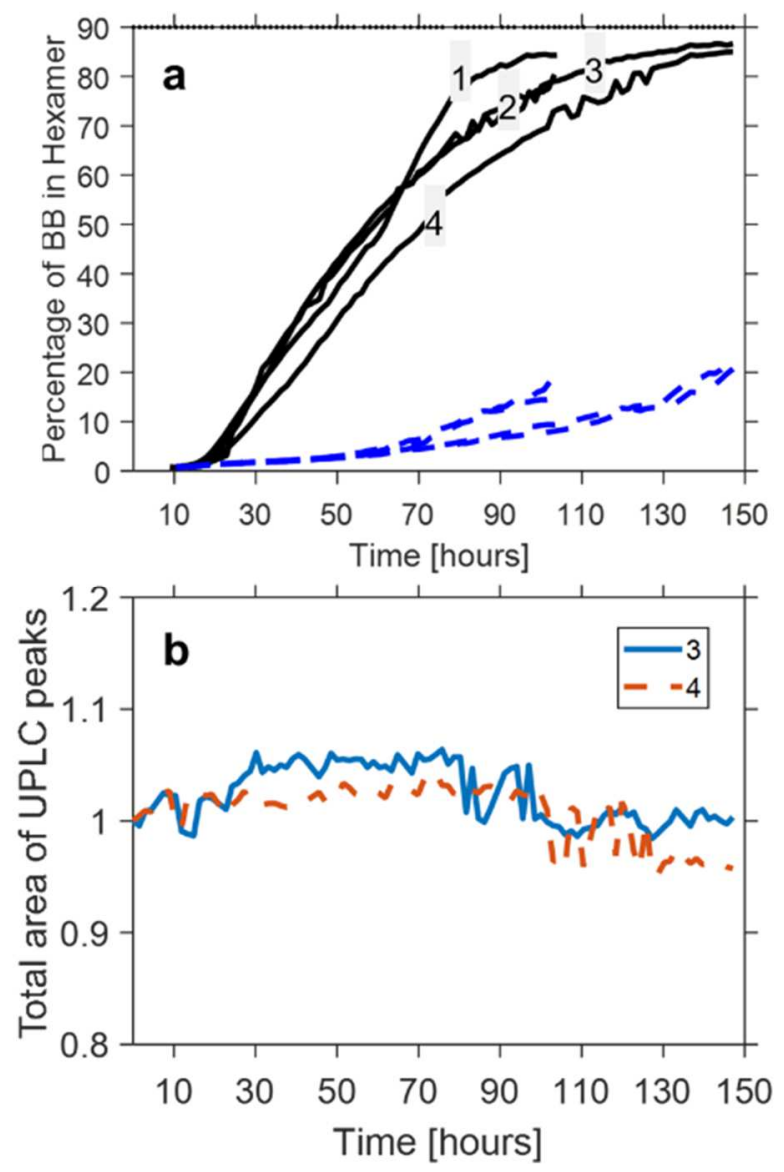

Fig. 3. Kinetics. (a) Hexamer emergence over time, given in percentage out of the initial buildingblock concentration. Black solid lines, stirred $1000 \mathrm{rpm}$. Blue broken lines, un-stirred. Black dots on the top of the figure indicate a time when a measurement was taken. For each condition, four repeats were performed. (b) Total area of all UPLC peaks detected by the UPLC software, relative to the initial total area (respectively $1.752 \times 107$ and $1.849 \times 107$ [arbitrary units]). 


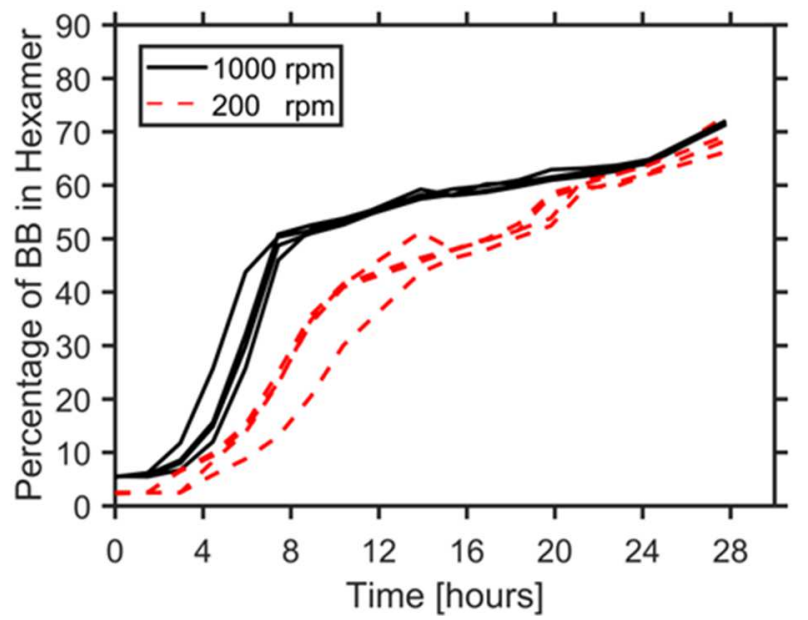

Fig. 4. Hexamer emergence over time under pre-oxidation (see experimental section). Time to reach 50\%: 8.166 \pm 0.857 hours (1000 rpm), 17.23 \pm 2.226 hours (200 rpm). Four repeats were conducted for each condition.

\section{Availability}

Instructions for re-producing and constructing the stirring device are available via the SI or from the corresponding author.

\section{CONCLUSIONS}

A device for the continuous stirring of samples whilst inside a UPLC system is presented and demonstrated. Such a device allows for the automation of experiments that require stirring and facilitates high frequency and reproducibility of UPLC injections. The latter may be particularly advantageous when studying and modelling complex dynamic chemistries ${ }^{11,12}$. It is our belief that such a device can be beneficial for other analytical laboratories around the globe.

In principle it is possible to amend the present design to fit to other chromatography machines and extend its capabilities. It is also possible to program the onboard microcontroller to allow for more complicated scenarios.

\section{ACKNOWLEDGMENTS}

O.M. is funded through the NWA StartImpuls. This work has been funded by the ERC, the NWO and the Dutch Ministry of Education, Culture and Science. We thank Andreas Hussain for discussions and Shraddha Shitut for making the Table of Contents artwork.

\section{Author Contributions}

O.M. designed the device, with input from J.O. and O.V. J.O. designed and performed experiments, with input from O.M. O.V constructed device. O.M. wrote manuscript draft. All authors wrote, read and approved the manuscript.

\section{Competing interests}

O.V. was hired for several assignments during the development of the device. 


\section{Data availability}

The data supporting this publication are available from the corresponding author upon request.

\section{REFERENCES}

1. Swartz ME. UPLC ${ }^{\mathrm{TM}}$ : An Introduction and Review. Journal of Liquid Chromatography \& Related Technologies 2007, 28(7-8): 1253-1263.

2. Lee CC, Nayak A, Sethuraman A, Belfort G, McRae GJ. A three-stage kinetic model of amyloid fibrillation. Biophys $J$ 2007, 92(10): 3448-3458.

3. Malakoutikhah M, Peyralans JJ, Colomb-Delsuc M, Fanlo-Virgos H, Stuart MC, Otto S. Uncovering the selection criteria for the emergence of multi-building-block replicators from dynamic combinatorial libraries. J Am Chem Soc 2013, 135(49): 18406-18417.

4. Carnall JM, Waudby CA, Belenguer AM, Stuart MC, Peyralans JJ, Otto S. Mechanosensitive selfreplication driven by self-organization. Science 2010, 327(5972): 1502-1506.

5. Colomb-Delsuc M, Mattia E, Sadownik JW, Otto S. Exponential self-replication enabled through a fibre elongation/breakage mechanism. Nat Commun 2015, 6: 7427.

6. Fisher DK, Gould PJ. Open-Source Hardware Is a Low-Cost Alternative for Scientific Instrumentation and Research. Modern Instrumentation 2012, 01(02): 8-20.

7. Meloni GN, Bertotti M. 3D printing scanning electron microscopy sample holders: A quick and cost effective alternative for custom holder fabrication. PLoS One 2017, 12(7): e0182000.

8. Pohanka M. Three-Dimensional Printing in Analytical Chemistry: Principles and Applications. Analytical Letters 2016, 49(18): 2865-2882.

9. Kitson PJ, Marshall RJ, Long D, Forgan RS, Cronin L. 3D printed high-throughput hydrothermal reactionware for discovery, optimization, and scale-up. Angewandte Chemie International Edition 2014, 53(47): 12723-12728.

10. Badamasi YA. The working principle of an Arduino. 2014 11th International Conference on Electronics, Computer and Computation (ICECCO); 2014: IEEE; 2014. p. 1-4.

11. Ashkenasy G, Hermans TM, Otto S, Taylor AF. Systems chemistry. Chem Soc Rev 2017, 46(9): 2543-2554.

12. Markovitch O, Krasnogor N. Predicting species emergence in simulated complex pre-biotic networks. PLoS One 2018, 13(2): e0192871. 